\title{
Networked Virtual Marionette Theater
}

\author{
Daisuke Ninomiya', Kohji Miyazaki ${ }^{1}$, Ryohei Nakatsu ${ }^{1,2}$ \\ 1 Kwansei Gakuin University, School of Science and Technology \\ 2-1 Gakuen, Sanda, 669-1337 Japan \\ \{aaz61232, miyazaki, nakatsu\}@kwansei.ac.jp \\ http:www.kwansei.ac.jp \\ ${ }^{2}$ National University of Singapore, Interactive \& Digital Media Institure \\ Block E3A, \#02-04, 7 Engineering Drive 1, Singapore 117574 \\ idmdir@nus.edu.sg \\ http://www.idmi.nus.edu.sg
}

\begin{abstract}
This paper describes a system that allows users to control virtual marionette characters based on computer graphics (CG marionette characters) with their hand and finger movements and thus perform a marionette theatrical play. The system consists of several subsystems, and each subsystem consists of a web camera and a PC. It can recognize a hand gesture of its user and transform it into a gesture of a CG marionette character. These subsystems are connected through the Internet, so they can exchange the information of the CG marionette character's movements at each subsystem and display the movements of all characters throughout the entire system. Accordingly, multiple users can join the networked virtual marionette theater and enjoy the marionette play together.
\end{abstract}

Keywords: Marionette, puppet, virtual theater, hand gesture, image recognition

\section{Introduction}

The culture of controlling puppets with the hands to perform theatrical play has been common throughout the world from ancient times. In Japan, there is a type of puppet theater called Bunraku, which arose about three hundred years ago [1][2]. In Europe, too, various kinds of puppet play have been performed and enjoyed. The puppet play using a puppet called a "marionette" has been the most popular variety [3]. Marionette play and puppets have become very popular in recent years, largely due to the movie called "Strings [4]" (Fig. 1). This paper describes a 
networked virtual marionette theater that is basically a distributed system consisting of several subsystems connected through the Internet. Each subsystem can recognize the hand and finger gestures of the person in front of its web camera and then transform them into the motions of a marionette character based on computer graphics (CG marionettes). Each subsystem exchanges the information of actions performed by its marionette character with such information from the other subsystems. The display of each subsystem shows a virtual scene where multiple marionette characters, each controlled by a different user, interact. Thus multiple users, even if they are in separate locations, can gather in a virtual marionette theater and perform a theatrical marionette play.

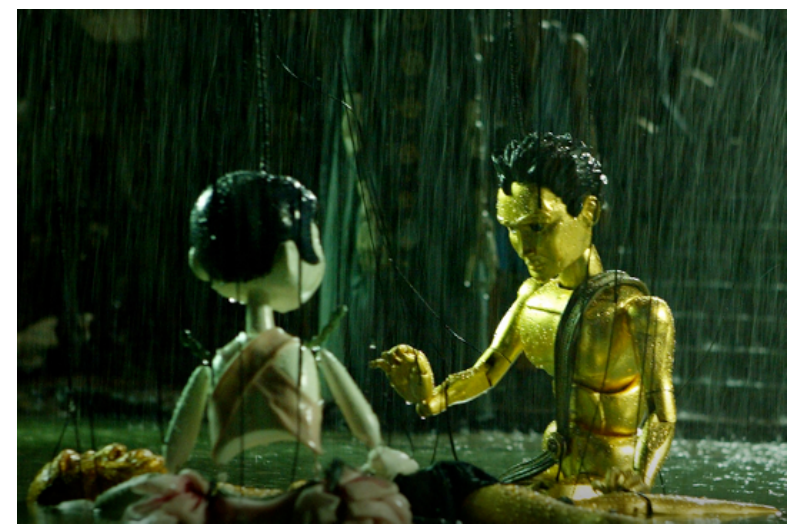

Fig. 1. A scene of "Strings"

\section{Related Works}

Technologies based on three-dimensional computer graphics have made tremendous progress in recent years. We can see photographically real CG objects and $\mathrm{CG}$ characters in movies and games. Furthermore, the technologies based on CG animation have also progressed rapidly. Animations of fluid [5] and the destruction of objects [6] have been studied. Moreover, the movements of a crowd based on an artificial-intelligence approach [7] and movements of humans based on inverse kinematics [8] have been proposed. Motion capture systems have been widely used for the control of CG characters [9]. Although the realization of human-like motions of CG characters has been eagerly pursued, the realization of marionette-like motions has seldom been studied. Since the movements of marionette characters are unique and have been loved by people throughout history, it is worth studying a system by which non-experts of marionettes can easily manipulate their movements and generate marionette-like behaviors using CG characters. 


\section{System Concept}

The following elements typically compose a marionette theater.

(1) Puppets called "marionettes"

(2) Speech of each puppet

(3) Scene settings

(4) Music

In a large performance, various kinds of marionette puppets appear and the scene settings are changed frequently, depending on the story's plot, and even a live orchestra is sometimes used to generate music. Therefore, even if people wanted to enjoy manipulating marionette puppets and creating theatrical play, it could be very difficult. On the other hand, if we introduced virtual marionette characters based on computer graphics instead of using real marionettes with physical bodies, it would become significantly easier for users to generate and change most of the above elements of marionettes, speech, backgrounds, and music. In addition, by developing a networked virtual marionette theater, multiple users, manipulating their own marionette characters, can gather in a virtual theater and let their virtual puppets interact with other puppets, thus creating the performance of a virtual theatrical play.

\section{System Structure}

\subsection{Overview}

The entire system is made from a group of subsystems connected through a network. The structure of the whole system is shown in Fig. 2, and the structure of each subsystem is illustrated in Fig. 3. Each subsystem consists of a PC and a web camera. The posture of a user's hand is captured by the web camera, and then hand-gesture recognition is carried out. Then the recognition result of a hand posture is reflected in the gestures of a CG marionette character. 


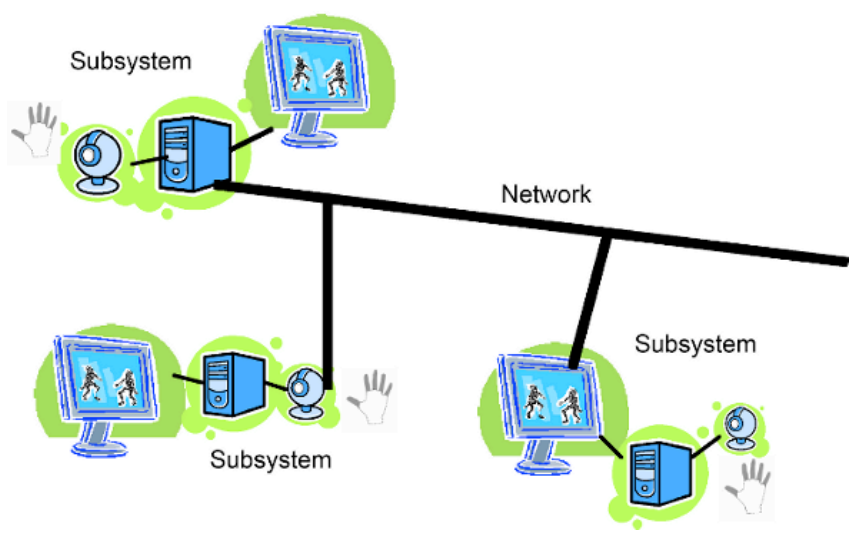

Fig. 2. Structure of entire system

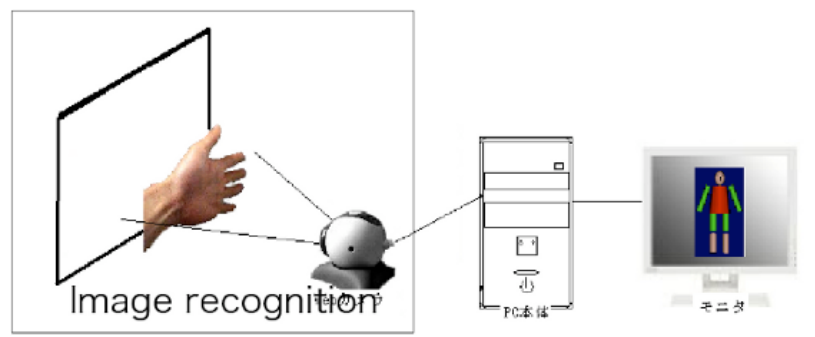

Fig. 3. Structure of subsystem

\subsection{Hand-gesture recognition}

In this section, a real-time hand-gesture recognition method is described for use in the recognition of a user's hand gesture for each subsystem [5]. There have been several research efforts on the real-time recognition of hand gestures [6][7]. Most of them use rather complicated systems such as multiple cameras. On the other hand, we tried to develop a simpler system using a single web camera. The recognition process consists of the following sub-processes. 


\subsubsection{Extraction of hand area (Fig. 4)}

Using the color information of a hand, the image area corresponding to a hand is extracted from the background. In this case, HSV information obtained by the transformation of RGB information is used. Then, using a median filter, the noise contained in the extracted image is deleted.

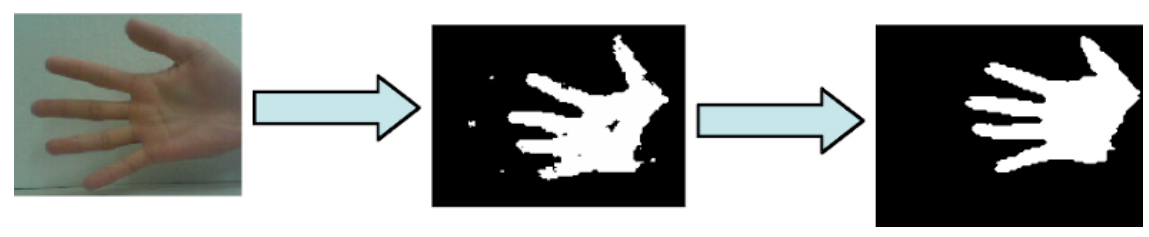

Fig. 4. Extraction of hand area

\subsubsection{Extraction of finger information using histogram}

The length of each finger is calculated by using simple histogram information. Figure 5 shows the information of a histogram corresponding to finger length. Depending on the angle of finger bending, the height of the histogram varies. This means that from the height information of the histogram, the bending angle of a finger can be calculated.

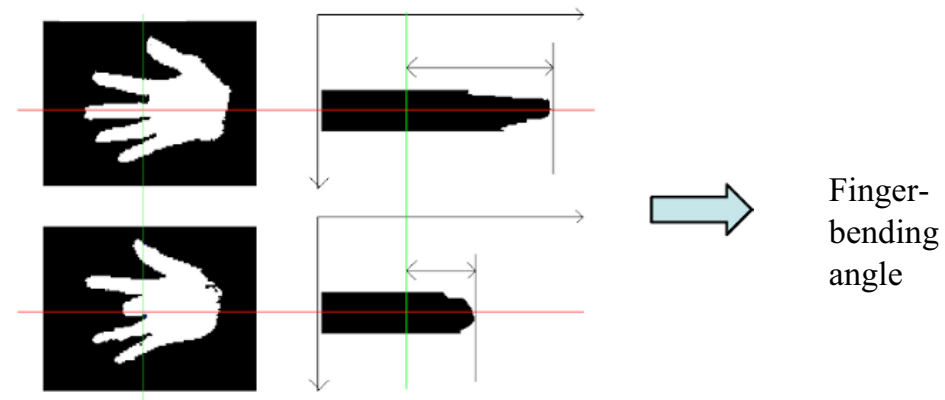

Histogram

Fig. 5. Extraction of finger-length information 


\subsubsection{Optimization of separating each finger's histogram}

Depending on the angle of each finger against the $\mathrm{x}$ axis (or $\mathrm{y}$ axis), it is sometimes difficult to clearly separate a histogram corresponding to each finger. Therefore, for the information extraction of each finger, rotation transformation is carried out to achieve the optimum separation of partial histograms corresponding to each finger.

\subsubsection{Bending-angle estimation of each finger}

Figure 5 also shows a comparison between two histograms varying with the bending angle of a finger. By comparing the length of a histogram to the original (longest) histogram when the bending angle is zero, the bending angle of the finger is calculated.

\subsection{Control of CG marionette}

Each finger is assumed connected to a certain part of a CG marionette through a virtual string. The relationship between five strings and the part of the marionette to which each string is attached is illustrated in Fig. 6. Here, $\mathrm{t} 1 \sim \mathrm{t} 5$ are virtual stings, and p1 p 8 are the parts composing the marionette model, where a1 $\sim$ a7 are joints of these parts. The bending angle of each finger calculated in the above process is reflected directly in the length of each string. In this way, the angle of each joint of the marionette, corresponding to p1, p2, p3, p4, p5, p6, p7, and p8, is determined. Therefore, by bending each of the five fingers appropriately, a user can control the motion and gestures of a virtual CG marionette.

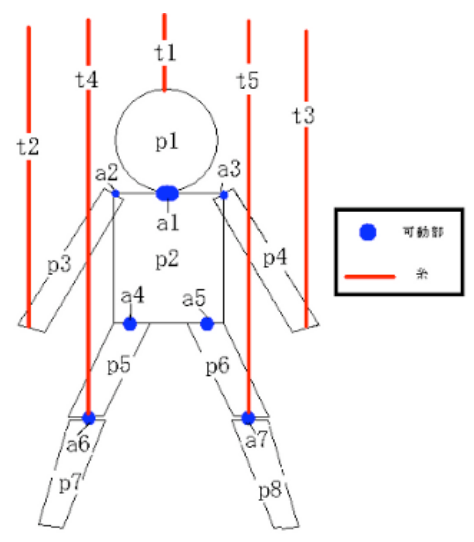

Fig. 6. Model of a virtual marionette 


\subsection{Background and CG characters}

We are planning a system that allows us to easily change scenes as well as characters, so we have developed various kinds of backgrounds and characters based on computer graphics. We are trying to develop an "Interactive Folktale System [8]" that offer users the ability to generate Japanese folktales as animation and to enjoy the interactions with creators of other characters in the system. Therefore, we have prepared various kinds of backgrounds and characters for our virtual marionette system. Figure 7 shows one of the marionette character in three different backgrounds.

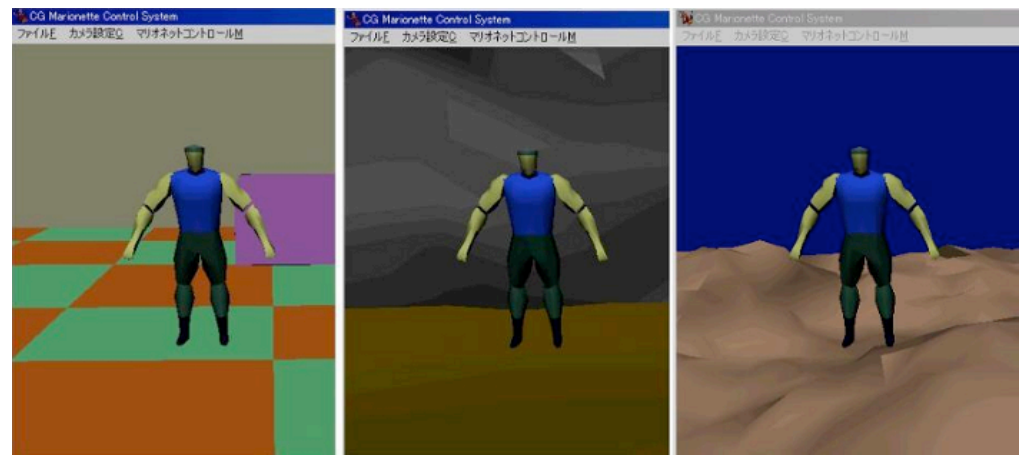

Fig. 7. Examples of virtual marionette characters

\subsection{Networked marionette theater}

The virtual marionette system we have developed as a first prototype toward the networked virtual marionette system consists of a hand-gesture recognition unit and an animation generation unit. This prototype system would work as a subsystem in the distributed system. In each subsystem, the recognition results of the other subsystems are shared. Furthermore, all of the CG characters and backgrounds are shared among these subunits. Using these recognition results as well as the CG characters and backgrounds, each subsystem can simultaneously create 
the same scene where multiple CG characters, each of which is controlled by its own subsystem, appear and behave in the same way.

\section{Evaluation of the System}

We have carried out an evaluation of a subsystem, which is the basis of the whole system and the instrument with which a user can control one virtual marionette character. We selected 20 students as subjects for this evaluation's tests. All of them know about marionette puppets but have never manipulated them. We asked them to manipulate both a real marionette puppet and a virtual CG marionette used in this system. After that we asked them several questions. The questions and the answers are summarized as follows.

(1) Is the movement of a virtual marionette "unique" compared with other CG characters?

Definitely Yes (4), Comparatively Yes (12), Neutral (4), Comparatively No (0), Definitely No (0)

(2) Is the movement of a virtual marionette "real"?

Definitely Yes (0), Comparatively Yes (1), Neutral (15), Comparatively No (4), Definitely No (0)

(3) Did you feel that your hand gestures were closely reflected in the movements of a virtual marionette?

Definitely Yes (0), Comparatively Yes (15), Neutral (3), Comparatively No (1), Definitely No (1)

From the first question, it is clear that $80 \%$ of the subjects said that there is some unique aspect in the movement of the virtual marionette. This means that the authors succeeded in their intention to develop a system in which the particular movement of a marionette is regenerated. For the second question, the fact that most of the subjects answered "neutral" indicates that the meaning of "real" is somewhat difficult for them to associate with the marionette's movement. For the third question, $75 \%$ of the subjects answered that the marionette correctly moved according to their hand gestures. These results show that the recognition method introduced here works very well and gives people the feeling that they are directly manipulating the virtual marionette characters. Moreover, they again expressed the feeling that the system successfully reproduced the particular movement of a marionette. 


\section{Conclusions}

In this paper, we proposed a system in which users can easily manipulate virtual marionette characters with their hand gestures. For the recognition of hand gestures, simple real-time hand-gesture recognition was realized by using histogram information of an extracted hand area. The recognition result is reflected in the movement of the marionette character by connecting each finger movement to a particular part of the virtual marionette by a virtual string. Furthermore, the concept of networked marionette theater was proposed in which several subsystems are connected by a network. Here, multiple users can perform theatrical marionette play by manipulating their own marionette characters. Finally, we carried out an evaluation test to assess the feasibility of a subsystem. By using twenty subjects and letting them manipulate both a physical marionette as well as a virtual one, we obtained evaluation results indicating that by using this virtual marionette system, even a non-expert of marionette manipulation can have the feeling of manipulating marionettes and thus can participate in a theatrical marionette performance.

For our further work, we need to improve the recognition accuracy of the handgesture recognition. Moreover, we need to develop adequate contents to refine the entire networked virtual marionette theater, and we also need to carry out an evaluation of the whole system by letting people use the system.

\section{References}

1. Keene, D. No and Bunraku. (1990). Columbia University Press.

2. http://www.lares.dti.ne.jp/bunraku/index.html

3. Currell D. Making and Manipulating Marionettes. (2004). The Crowood Press Ltd.

4. http://www.futuremovies.co.uk/review.asp?ID=319

5. Stam, J. and Fiume, E. Depicting Fire and Other Gaseous Phenomena Using Diffusion Process. (1995). In Proceedings of SIGGRAPH'95.

6. O' Brien, J. F. and Hodgins J. K. Graphical modeling and animation of brittle fracture. (1999). In Proceedings of SIGGRAPH'99.

7. Courty, N. Fast Crowd. (2004). In Proceedings of SIGGRAPH ' 2004.

8. Boulic N., Thalmann, M. and Thalmann, D. A GLOBAL HUMAN WALKING MODEL WITH REAL-TIME KINEMATIC PERSONIFICATION. (1990). The Visual computer, pp. 344-358.

9. Lee, J. and Lee, K. H. Precomputing avatar behavior from human motion data. (2004). Graphical Models, Vol. 68, No. 2, pp. 158-174.

10. Ninomiya, D., Miyazaki, K. and Nakatsu, R. Study on the CG Marionette Control Based on the Hand Gesture Recognition. (2006). Annual Meeting of Game Society of Japan (in Japanese).

11. Ng, C. W. Real-time gesture recognition system and application. (2002). Image and Vision Computing, pp. 20. 
12. Utsumi, A., Ohya, J. and Nakatsu, R. Multiple-camera-based Multiple-hand-gesturetracking. (1999). Transaction of Information Processing Society of Japan, Vol. 40, No. 8, pp. 3143-3154 (in Japanese).

13. Miyazaki, K., Nagai, Y., Wama, T. and Nakatsu, R. Concept and Construction of an Interactive Folktale System. (2007). Entertainment Computing - ICEC2007, Springer LNCS 4740, pp. 162-170. 